\title{
Liver toxicity and risk of discontinuation in HIV/hepatitis C virus-coinfected patients receiving an etravirine-containing antiretroviral regimen: influence of liver fibrosis
}

\author{
JL Casado, ${ }^{1}$ A Mena, ${ }^{2}$ S Bañón, ${ }^{1}$ A Castro, ${ }^{2}$ C Quereda, ${ }^{1}$ A Moreno,,${ }^{1} \mathrm{~J}$ Pedreira, ${ }^{2}$ \\ and $\mathrm{S}$ Moreno ${ }^{1}$ \\ ${ }^{1}$ Department of Infectious Diseases, Ramon y Cajal Hospital, Madrid, Spain \\ ${ }^{2}$ Department of Internal Medicine, INIBIC-Complexo Universitario Hospitalario, A Coruña, Spain
}

\begin{abstract}
Objectives. The aim of the study was to establish the risk of liver toxicity in HIV/hepatitis C virus (HCV)coinfected patients receiving etravirine, according to the degree of liver fibrosis.

Methods. A prospective cohort study of $211 \mathrm{HIV}$-infected patients initiating an etravirine-containing regimen was carried out. HCV coinfection was defined as a positive HCV RNA test, and baseline liver fibrosis was assessed by transient elastography. Hepatotoxicity was defined as clinical symptoms, or an aspartate aminotransferase (AST) or alanine aminotransferase (ALT) value $>5$-fold higher than the upper limit of normal if baseline values were normal, or 3.5-fold higher if values were altered at baseline.

Results. Overall, 145 patients $(69 \%)$ were HCV coinfected, with a lower nadir $(165$ versus 220 cells $/ \mu \mathrm{L}$, respectively; $p=0.03$ ) and baseline (374 versus 498 cells $/ \mu \mathrm{L}$, respectively; $p=0.04)$ CD4 count than monoinfected patients. Etravirine was mainly used with two nucleoside reverse transcriptase inhibitors (129; $61 \%$ ) or with a boosted protease inhibitor (PI) (28\%), with no significant differences according to HCV serostatus. Transient elastography in 117 patients $(81 \%)$ showed a median (range) stiffness value of 8.25 (3.5-69) $\mathrm{kPa}$, with fibrosis stage 1 in 43 patients (37\%) and fibrosis stage 4 in 28 patients (24\%). During an accumulated follow-up time of 449.3 patient-years (median 548 days), only one patient with advanced fibrosis $(50.8 \mathrm{kPa})$ had grade $3-4$ liver toxicity $(0.7 \%)$. Transaminases changed slightly, with no significant differences compared with baseline fibrosis, and nine and six patients had grade 1 and 2 transaminase increases, respectively. Also, $\mathrm{HCV}$ coinfection was not associated with a higher risk of discontinuation (25\% discontinued versus $21 \%$ of monoinfected patients; $p=0.39$, log-rank test) or virological failure ( $8 \%$ versus $12 \%$, respectively; $\mathrm{p}=0.4$ ).

Conclusions. Our data suggest that etravirine is a safe option for HIV/HCV-coinfected patients, including those with significant liver fibrosis.
\end{abstract}

Keywords

Etravirine, Hepatotoxicity, Liver fibrosis, Nonnucleoside reverse transcriptase inhibitor, Transient elastography 


\section{Introduction}

Etravirine (ETR), a second-generation nonnucleoside reverse transcriptase inhibitor (NNRTI), has demonstrated good tolerability and efficacy in treatment-naïve and experienced patients [1,2]. In those studies, discontinuations because of adverse events were rare, representing less than $10 \%$ of cases. In particular, there was a low incidence of grade 3-4 transaminase elevation and drug discontinuation because of liver-related adverse events, which were reported in $3 \%$ and $8 \%$ of patients, respectively [1,2]. However, data on liver safety in HIV/hepatitis C virus (HCV) coinfection were limited, as in both studies no more than $11 \%$ and $13 \%$ of patients were $\mathrm{HCV}$ coinfected, respectively, and patients with advanced liver disease are excluded from clinical trials.

Thus, the risk of liver toxicity in HIV/HCV-coinfected patients receiving ETR in the clinical setting is not fully established. It is particularly important to establish this risk, given that HCV coinfection is the most consistent risk factor for antiretroviral-associated hepatotoxicity [3]. Previous studies with other drugs of the same class have shown an incidence of liver toxicity of 3$20 \%$, with nevirapine having a higher risk than efavirenz in coinfected patients [4].

Furthermore, the risk of liver toxicity in coinfected patients with significant fibrosis or cirrhosis receiving ETR has not been established. Our group has previously demonstrated that the degree of liver fibrosis determines the risk of developing liver toxicity. Thus, the use of nevirapine or a ritonavir-boosted protease inhibitor $(\mathrm{PI} / \mathrm{r})$ was associated with different rates of toxicity according to whether mild or severe liver fibrosis was present [5].

Therefore, it is necessary to determine the risk of hepatotoxicity with the use of a new NNRTI such as ETR in HIV/HCV-coinfected patients with different grades of liver fibrosis in the clinical setting, this is important, as ETR has become an option for patients who need anti-HCV therapy [6]. In addition, patients coinfected with $\mathrm{HIV} / \mathrm{HCV}$ also are more likely to discontinue therapy because of toxicity [7].

Thus, the primary aim of this study was to determine the liver safety of highly active antiretroviral therapy (HAART) including ETR in HIV/HCV-coinfected patients, considering the stage of liver fibrosis, in terms of both liver enzyme elevation and regimen discontinuation, in comparison with patients without HCV infection.

\section{Methods}

This was a prospective cohort study that included patients attending two different specialized HIV units. Since 2010, patients initiating treatment with ETR were included if they met the following criteria: (i) initiation of antiretroviral therapy (ART) that included ETR; (ii) age $>18$ years; and (iii) demonstration of the presence (or absence) of chronic HCV infection as confirmed by HCV RNA in serum by polymerase chain reaction (PCR). Patients were excluded if they had other liver diseases, or alcohol consumption $>50 \mathrm{~g} /$ day. Clinical visits and blood tests were carried out at baseline, at month 1 , at month 3 and every 3 months thereafter. The study was designed and performed according to the Helsinki declaration and was approved by the Ethics Committee of our hospital (EC 150/06).

Patients were followed up until the end of July 2014, ETR discontinuation, or the development of liver toxicity, whichever occurred first. For patients who started treatment for HCV infection during follow-up, the date of end of follow up was the beginning of interferon-based therapy, since it was assumed a change in the risk of developing toxicity [8].

Changes in transaminase levels were classified using the Division of AIDS grading table [9]. Thus, in patients with normal baseline levels of alanine aminotransferase (ALT) and aspartate aminotransferase (AST), values of ALT or AST 5-10 times the upper limit of normal (ULN) were 
considered to indicate liver toxicity grade 3, while grade 4 was defined as ALT or AST values 10 times the ULN. When patients presented an increased baseline ALT or AST level, an elevation of between 3.5- and 5-fold was considered to indicate liver toxicity grade 3, and an elevation $>5$-fold to indicate grade 4 . Bilirubin levels were not considered in the definition of toxicity.

Baseline liver fibrosis was assessed using hepatic transient elastography (FibroScan®; Echosense, Paris, France). Briefly, the stiffness of the liver tissue was determined by ultrasound in the morning, after an overnight fast, as has been previously described [10]. According to Castera et al. [11], we classified liver fibrosis using a stiffness cutoff of $7.2 \mathrm{kPa}$ for fibrosis stage $2,9.5 \mathrm{kPa}$ for fibrosis stage 3, and $12.5 \mathrm{kPa}$ for fibrosis stage 4 . For example, this cutoff value of $12.5 \mathrm{kPa}$ had a positive and negative predictive value of $77 \%$ and $95 \%$, respectively, for the diagnosis of fibrosis stage 4 or cirrhosis.

The primary endpoint was hepatotoxicity, as defined above. The secondary outcomes were changes in transaminase levels and rate of discontinuation, according to $\mathrm{HCV}$ coinfection and liver fibrosis.

Continuous variables were analysed using Student's $t$-test for normal distributions and the Mann-Whitney $U$-test otherwise. Categorical variables were analysed with the $\chi^{2}$ test or Fisher's test, where applicable. For repeated transaminase measurements, the Wilcoxon signed rank test for continuous measurements was applied. The Kaplan-Meier method was used to assess the rate of discontinuation, and the influence of $\mathrm{HCV}$ coinfection was assessed using the log-rank test. All $P$ values $<0.05$ were considered statistically significant.

\section{Results}

Overall, 211 patients received an ETR-based regimen and were included in the study. Of these, 145 (69\%) were HCV coinfected. The baseline characteristics of all patients and those with HCV infection are shown in Table 1. As expected, HCV-coinfected patients had a higher prevalence of former injecting drug use as a risk practice and a longer time on HAART. Patients were followed for a median time of 548 days (interquartile range 256-1128 days). During follow-up, 50 patients (24\%) discontinued ETR, in 20 cases because of virological failure. Mild or moderate rash was observed in 12 patients, five of whom discontinued ETR. The rate of discontinuation in the group of HCV-coinfected patients was similar to that in $\mathrm{HCV}$-uninfected patients (25 versus $21 \%$; $P=0.39$; log-rank test) 
Table 1. Baseline characteristics of patients according to HIV/hepatitis C virus (HCV) coinfection

\begin{tabular}{|c|c|c|c|c|}
\hline Variable & $\begin{array}{l}\text { Overall } \\
(n=211)\end{array}$ & $\begin{array}{l}\text { HIV/HCV coinfection } \\
\quad(n=145 ; 69 \%)\end{array}$ & $\begin{array}{l}\text { HIV monoinfected } \\
\quad(n=66 ; 31 \%)\end{array}$ & $P$-value \\
\hline Age (years) [mean (range)] & $44(32-68)$ & $48(36-68)$ & $42(28-55)$ & 0.09 \\
\hline Sex male $[n(\%)]$ & $143(68)$ & $93(64)$ & $50(76)$ & 0.16 \\
\hline Former IDU $[n(\%)]$ & $133(63)$ & $130(90)$ & $3(5)$ & $<0.01$ \\
\hline $\begin{array}{l}\text { Nadir CD4 count (cells } / \mu \mathrm{L})[\text { median } \\
(\mathrm{IQR})]\end{array}$ & $185(97-287)$ & $165(88-252)$ & $220(97-349)$ & 0.03 \\
\hline $\begin{array}{l}\text { Time on HAART prior to ETR (months) } \\
\text { [median (IQR)] }\end{array}$ & $\begin{array}{l}155.3(52.7- \\
167.8)\end{array}$ & $166.3(112-197)$ & $57.4(9.7-140.3)$ & $<0.01$ \\
\hline $\begin{array}{l}\text { CD } 4 \text { count at inclusion }(\text { cells } / \mu \mathrm{L})[\text { median } \\
(\mathrm{IQR})]\end{array}$ & $400(221-678)$ & $374(215-672)$ & $498(269-770)$ & 0.04 \\
\hline $\begin{array}{l}\text { HIV RNA level } \leq 50 \text { copies } / \mathrm{mL} \text { at } \\
\text { inclusion }[n(\%)]\end{array}$ & $137(65)$ & $85(59)$ & $52(79)$ & 0.03 \\
\hline \multicolumn{5}{|l|}{ ETR plus $[n(\%)]:$} \\
\hline 2 NRTIs & $129(61)$ & $89(61)$ & $40(60)$ & \\
\hline $\mathrm{PI} / \mathrm{r}$ & $39(19)$ & $27(19)$ & $12(18)$ & 0.7 \\
\hline $\mathrm{PI} / \mathrm{r}+\mathrm{RTG}$ & $20(9)$ & $13(9)$ & $7(11)$ & \\
\hline $\mathrm{RTG} \pm \mathrm{NRTI}$ & $23(11)$ & $16(11)$ & $7(11)$ & \\
\hline \multicolumn{5}{|l|}{ Reasons for ETR initiation [ $n(\%)]$} \\
\hline Toxicity/intolerance & $141(67)$ & $93(64)$ & $48(73)$ & 0.29 \\
\hline Virological failure & $70(33)$ & $52(36)$ & $18(27)$ & \\
\hline Virological failure with ETR [ $n(\%)]$ & $20(10)$ & $12(8)$ & $8(12)$ & 0.45 \\
\hline Rash with ETR $[n(\%)]$ & $12(6)$ & $7(5)$ & $5(8)$ & 0.2 \\
\hline ETR discontinuation $[n(\%)]$ & $50(24)$ & $36(25)$ & $14(21)$ & 0.6 \\
\hline Time on ETR (days) [median (IQR)] & $\begin{array}{l}548(256- \\
1128)\end{array}$ & $502(233-925)$ & $673(317-1292)$ & 0.1 \\
\hline \multicolumn{5}{|l|}{$\begin{array}{l}\text { Transient elastography at baseline }[n \\
(\%)]^{*}\end{array}$} \\
\hline Fibrosis stage 1 & & $43(37)$ & & \\
\hline Fibrosis stage 2 & & $29(25)$ & & \\
\hline Fibrosis stage 3 & & $17(14)$ & & \\
\hline Fibrosis stage 4 & & $28(24)$ & & \\
\hline
\end{tabular}

*Available in $117 \mathrm{HIV} / \mathrm{HCV}$-coinfected patients.

IDU, injecting drug use; IQR, interquartile range; HAART, highly active antiretroviral therapy; ETR, etravirine; NRTI, nucleoside reverse transcriptase inhibitor; PI/r, protease inhibitor boosted with ritonavir; RTG, raltegravir; TE, transient elastography.

ETR was used with two nucleoside reverse transcriptase inhibitors (NRTIs) in $61 \%$ of patients (129 patients: tenofovir plus emtricitabine in 90 patients, and abacavir plus lamivudine in 39 patients), with a boosted PI (mostly darunavir) in 19\%, with darunavir plus raltegravir in $9 \%$, and with raltegravir \pm one NRTI (PI-sparing regimen) in $11 \%$ (23 patients).

During an accumulated follow-up time of 449.3 patient-years, only one patient, who had fibrosis stage 4 (stiffness value $50.8 \mathrm{kPa}$ ) and started a regimen with tenofovir/emtricitabine plus ETR plus darunavir/ritonavir, developed liver toxicity grade $3-4(0.7 \%)$. Transaminase (ALT value) changes for mono- and coinfected patients are shown in Fig. 1a. Also, there were six and nine cases of grade 2 and 1 transaminase elevations, respectively, in the HCV-coinfected population. 

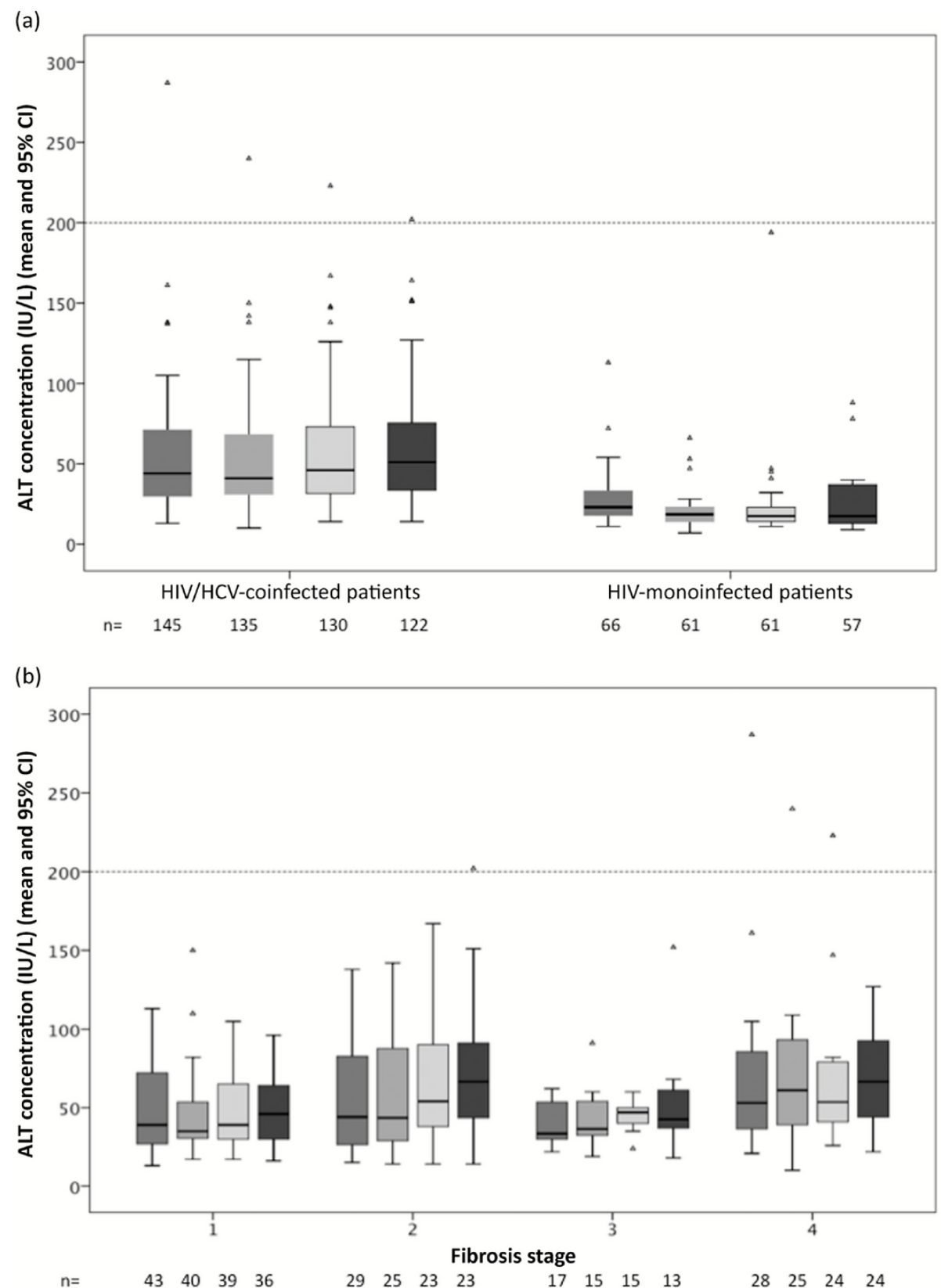

Figure 1. Changes in alanine aminotransferase (ALT) concentration during the first year after etravirine initiation (at baseline and months 3, 6 and 12), according to (a) hepatitis C virus (HCV) serostatus and (b) the degree of liver fibrosis. CI, confidence interval.

Liver fibrosis measurements obtained by transient elastography were available in 117 patients ( $81 \%$ of $\mathrm{HCV}$-coinfected patients). The median (range) stiffness value was $8.25(3.5-69) \mathrm{kPa}$. Fibrosis stage 4 was observed in $24 \%$ of patients (28 patients), with $37 \%$ of patients having fibrosis stage 1. Transaminase changes according to liver fibrosis stage were not significant (Fig. 1b). In patients with cirrhosis, there were no hepatic decompensations or other liver-related complications during the follow-up period. Discontinuation was similar according to liver fibrosis stage $(19,20,27$ and $21 \%$ for fibrosis stages 1 to 4 , respectively; $P=0.3)$. 


\section{Discussion}

This cohort study is the largest to investigate, in a clinical setting, the risk of liver toxicity with ETR in HIV-infected patients with chronic HCV coinfection. We found that the risk of toxicity was low. This finding was obtained irrespective of the degree of liver fibrosis and other drugs present in the regimen. There were few changes in transaminases among HIV/HCV-coinfected patients after starting an ETR-based regimen, and transaminase values in most patients remained within the baseline range observed before initiation of treatment with ETR.

This low incidence of liver toxicity contrasts with that observed in HIV/HCV-coinfected patients receiving HAART in previous studies. Even in patients receiving other well-tolerated drugs, such as raltegravir or maraviroc, liver events were observed in 5-10\% of patients [12,13], and a higher rate was found in patients receiving nevirapine-, efavirenz- or PI/r-based regimens [14]. Liver safety analyses for $\mathrm{PI} / \mathrm{r}$ in clinical trials in $\mathrm{HIV} / \mathrm{HCV}$-coinfected patients have shown an incidence of grade 3-4 transaminase elevations of between 6 and $15 \%$.

Our data are in accordance with those obtained in a subgroup analysis of the DUET studies, including only patients with HCV coinfection, in which liver toxicity in the ETR arm was similar to that observed in the placebo arm [15]. More recently, in a similar study in a clinical setting, Lopez-Cortés et al. [16] reported no cases of liver toxicity grade 3-4 in 103 patients with chronic $\mathrm{HCV}$ infection receiving ETR plus two NRTIs, and $<1 \%$ of patients with grade 2 increases in transaminases.

Importantly, the discontinuation rate among HIV/HCV-coinfected individuals starting HAART ranged from 4 to $13 \%$ for $\mathrm{PI} / \mathrm{r}$ - or nonnucleoside reverse transcriptase inhibitor (NNRTI)-based regimens. In a recent study, Grint et al. reported a $44 \%$ increased risk of antiretroviral drug discontinuation for HCV viraemic patients, and an even higher (59\%) increased risk for patients receiving NNRTIs, mostly nevirapine or efavirenz [7]. A hyaluronic acid concentration of $>100 \mathrm{ng} / \mathrm{mL}$, a marker of liver fibrosis, was associated with a $37 \%$ increased risk of antiretroviral drug discontinuation. In our study, the rate of discontinuation was not increased in HCV-coinfected patients receiving ETR, even when only patients with a more accurate determination of advanced fibrosis, obtained using transient elastography, were considered.

In addition, liver fibrosis did not increase significantly the rate of toxicity. The presence of cirrhosis may cause metabolic alterations and lead to overexposure to antiretroviral drugs. Our results suggest that ETR can be considered relatively safe in patients with cirrhosis. Only one coinfected patient with advanced cirrhosis starting a regimen with ETR plus boosted darunavir had toxicity. However, the stiffness cutoff value used for cirrhosis was low $(12.5 \mathrm{kPa})$, a threshold that provides a high negative predictive value $(95 \%)$ but a relatively low positive predictive value (77\%). Thus, although some of our patients with fibrosis stage 4 or cirrhosis could be classified as having fibrosis stage 3 if a different criterion is used [17], most of our patients had moderate or advanced liver fibrosis (63\% had fibrosis stage 2 or higher).

The underlying reason for this low risk of hepatotoxicity has not been fully established. In a phase I pharmacokinetic study, no clinically relevant differences were observed between patients with mild or moderate hepatic impairment and healthy matched HIV-negative subjects with regard to the pharmacokinetics of ETR, and no dose adjustment of ETR was necessary in patients with mild or moderate hepatic impairment [18]. Also, ETR oral clearance seemed not to be affected by hepatitis B virus coinfection in a pharmacokinetic study, and no relationship was found between ETR pharmacokinetics and either efficacy or safety [19]. However, ETR produces lower hepatic cell induction than other NNRTIs, a fact that could explain our results [20].

This low risk of hepatotoxicity could permit the safe use of ETR while patients are on HCV therapy including drugs such as telaprevir, since the concomitant use of both drugs has been demonstrated to be safe and effective previously [6]. However, there are few data about drug - 
drug interactions between ETR and new anti-HCV agents. In vivo, ETR is a substrate for, and a weak inducer of, the hepatic cytochrome P450 (CYP) isoenzyme 3A4 and a substrate and weak inhibitor of CYP2C9 and CYP2C19. Also, it is a weak inhibitor of P-glycoprotein (P-gp) [19]. Thus, hypothetically, ETR could decrease levels of some new HCV NS3/4a protease inhibitors (simeprevir, paritaprevir, asunaprevir and grazoprevir), NS5A inhibitors (daclatasvir, ombutasvir and elbasvir) and NS5B inhibitors (dasabuvir), which are metabolized by CYP3A4 and/or transported by P-gp [21]. However, sofosbuvir and ledipasvir are not influenced by a potent CYP3A4 inducer such as efavirenz, and daclatasvir requires only increased doses. Therefore, our knowledge about interactions between ETR and anti-HCV agents needs to be improved.

The main limitation of our study is the cohort design. Thus, there are no comparative data on the use of other drugs in the same patients which could put into perspective the risk of ETR use. Furthermore, visits are more frequent in clinical trials, allowing the detection of more laboratory abnormalities. However, patients were followed in a clinical schedule. It is therefore unlikely that the prevalence of clinically relevant hepatotoxic events was underestimated. In addition, fibrosis assessment was not possible in all HCV-coinfected patients. However, nearly $90 \%$ of HCVcoinfected patients could be classified in terms of fibrosis, and can therefore be regarded as representative of the whole study population. Finally, fibrosis was assessed using transient elastography, not liver biopsy. Nevertheless, it has been demonstrated that there is a high correlation between liver biopsy and transient elastometry results [10].

In conclusion, the liver tolerance of ETR in $\mathrm{HIV} / \mathrm{HCV}$-coinfected patients in real-life conditions of use was high, even in patients with significant liver fibrosis. Therefore, ETR seems to be a safe option for coinfected patients.

\section{Author contributions}

JLC and AM designed the study, were responsible for patient enrolment and data analysis, and drafted and finalized the manuscript. SB, CQ, AM, JDP and SM were responsible for patient enrolment and taking measurements, clinically followed up patients, and helped to write the manuscript. All coauthors revised the manuscript and read and approved the final version.

\section{Conflicts of interest}

The study was supported by internal funding. No author has a commercial or other association that may pose a conflict of interest. All research was conducted within the guidelines of ethical principles, and local legislation.

\section{References}

Gazzard B, Duvivier C, Zagler C et al. Phase 2 double-blind, randomized trial of etravirine versus efavirenz in treatment-naive patients: 48-week results. AIDS 2011; 25: 2249-2258.

Katlama C, Clotet B, Mills A et al. Efficacy and safety of etravirine at week 96 in treatment-experienced HIV type-1-infected patients in the DUET-1 and DUET-2 trials. Antivir Ther 2010; 15: 1045-1052.

Núñez M. Hepatotoxicity of antiretrovirals: incidence, mechanisms and management. J Hepatol 2006; 44 (1 Suppl): S132-S139.

Rivero A, Mira JA, Pineda JA. Liver toxicity induced by non-nucleoside reverse transcriptase inhibitors. J Antimicrob Chemother 2007; 59: 342-346.

Aranzabal L, Casado JL, Moya J et al. Influence of liver fibrosis on highly active antiretroviral therapyassociated hepatotoxicity in patients with HIV and hepatitis C virus coinfection. Clin Infect Dis 2005; 40: 588-593.

Ramirez ML, Vargas FX, Gonzalez-Garcia J et al. Etravirine-based antiretroviral therapy in HIV/hepatitis C virus coinfected advanced fibrosis patients receiving triple therapy against hepatitis $\mathrm{C}$ virus with telaprevir. AIDS 2014; 28: 2487-2489. 
Grint P, Peters L, Rockstroh J et al. Increased incidence of antiretroviral drug discontinuation among patients with viremic hepatitis $C$ virus coinfection and high hyaluronic acid, a marker of liver fibrosis. AIDS 2014; 28: 577-587.

Labarga P, Soriano V, Vispo ME et al. Hepatotoxicity of antiretroviral drugs is reduced after successful treatment of chronic hepatitis C in HIV-infected patients. J Infect Dis 2007; 196: 670-676.

Division of AIDS (DAIDS). Table for grading the severity of adult and pediatric adverse events. 2004. (Clarification August 2009). Available at: http://www.niaid.nih.gov (accessed 30 July 2014).

Sánchez-Conde M, Montes-Ramírez ML, Miralles P et al. Comparison of transient elastography and liver biopsy for the assessment of liver fibrosis in HIV/hepatitis C virus-coinfected patients and correlation with noninvasive serum markers. J Viral Hepat 2010; 17: 280-286.

Castera L. Transient elastography and other noninvasive tests to assess hepatic fibrosis in patients with viral hepatitis. J Viral Hepat 2009; 16: 300-314.

Vispo E, Mena A, Maida I et al. Hepatic safety profile of raltegravir in HIV-infected patients with chronic hepatitis C. J Antimicrob Chemother 2010; 65: 543-547.

Lazzarin A, Than S, Valluri SR, Heera J, Mukwaya G. Safety profile of maraviroc in patients coinfected with HIV-1 and hepatitis B or C included in the maraviroc expanded access program. HIV Clin Trials 2012; 13: 83-89.

Macias J, Neukam K, Mallolas J et al. Liver toxicity of initial antiretroviral drug regimens including two nucleoside analogs plus one non-nucleoside analog or one ritonavir-boosted protease inhibitor in HIV/HCV-coinfected patients. HIV Clin Trials 2012; 13: 61-69.

Clotet B, Clumeck N, Katlama C, Nijs S, Witek J. Safety of etravirine in HIV-1 hepatitis B or/and C coinfected patients: pooled 96-weeks results from the phase III DUET trials. J Antimicrob Chemother 2010; 65: 2450-2454.

Lopez-Cortés LF, Viciana P, Girón-Gonzalez JA et al. Clinical and virological efficacy of etravirine plus two active nucleoside analogs in an heterogenous HIV-infected population. PLoS ONE 2014; 9: e97262.

Stebbing J, Farouk L, Panos G et al. A meta-analysis of transient elastography for the detection of hepatic fibrosis. J Clin Gastroenterol 2010; 44: 214-219.

Schöller-Gyüre M, Kakuda TN, De Smedt G et al. Effects of hepatic impairment on the steady-state pharmacokinetics of etravirine $200 \mathrm{mg}$ BID: an open-label, multiple-dose, controlled Phase I study in adults. Clin Ther 2010; 32: 328-337.

Kakuda TN, Wade JR, Snoeck E et al. Pharmacokinetics and pharmacodynamics of the non-nucleoside reverse-transcriptase inhibitor etravirine in treatment-experienced HIV-1-infected patients. Clin Pharmacol Ther 2010; 88: 695-703.

Yanakakis LJ, Bumpus NN. Biotransformation of the antiretroviral drug etravirine: metabolite identification, reaction phenotyping, and characterization of autoinduction of cytochrome P450-dependent metabolism. Drug Metab Dispos 2012; 40: 803-814.

Back D, Else L. The importance of drug-drug interactions in the DAA era. Dig Liver Dis 2013; 45 (Suppl 5): S343-S348. 